Gynäkologische Endokrinologie 2011 · 9:5-6 DOI 10.1007/s10304-010-0406-6

Online publiziert: 11. Februar 2011

(c) Springer-Verlag 2011

\author{
K. Diedrich ${ }^{1} \cdot$ T. Strowitzki ${ }^{2}$ \\ ${ }^{1}$ Klinik für Frauenheilkunde und Geburtshilfe, Universitätsklinikum \\ Schleswig-Holstein, Campus Lübeck \\ ${ }^{2}$ Abteilung für Gynäkologische Endokrinologie und Fertilitätsstörungen, \\ Universitäts-Frauenklinik, Heidelberg
}

\title{
Nobelpreis für Professor Robert G. Edwards
}

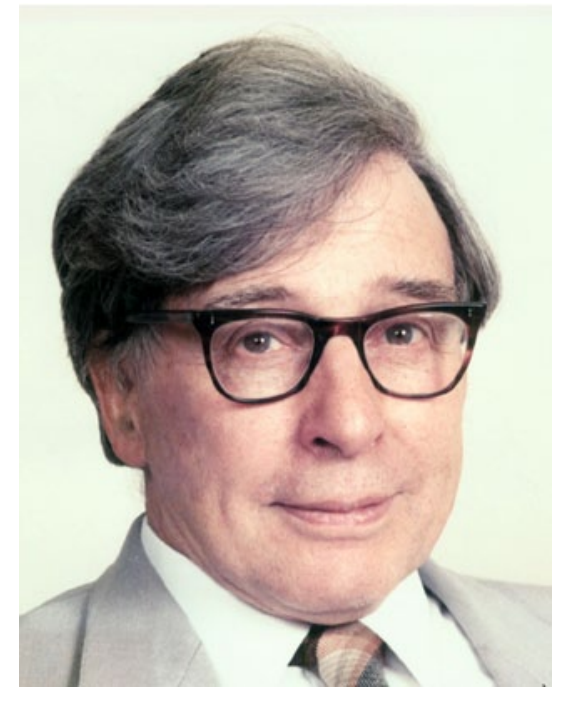

erte es nur kurze Zeit, bis der erste Inseminationsversuch gelang. Doch waren die Embryonen anfänglich nicht ausreichend entwicklungsfähig. Steptoe und Edwards gaben nicht auf, obwohl sie gegen erhebliche Widerstände kämpfen und ohne gesicherte Finanzierung arbeiten mussten. „Ich werde niemals den Tag vergessen“, erinnerte sich Edwards im Jahr 2008, „Ich blickte in das Mikroskop und was ich sah, war eine menschliche Blastozyste. Es war wunderbar. “ Das war 1972. Trotzdem dauerte es noch 6 Jahre bis 1978 Louise Brown geboren wurde, das erste durch In-vitroFertilisation (IVF) gezeugte Kind. Edwards und Steptoe arbeiteten eng zusammen bis zu Steptoes Tod 1988.

Im Jahr 1978 lernte ich selbst Robert Edwards auf einem Kongress in Japan kennen. In enger Zusammenarbeit mit den Kollegen in Großbritannien wurden auch in Europa und Deutschland Zentren für Reproduktionsmedizin gegründet. Die Standorte der damaligen 3 deutschen Zentren lagen in Erlangen unter Leitung von Siegfried Trotnow, in Lübeck unter Dieter Krebs und in Kiel unter Lieselotte Mettler. Das erste IVF-Kind Deutschlands wurde 1982 in Erlangen geboren, im darauf folgenden Jahr kam ein IVF-Kind in Lübeck zur Welt.

Als 1984 der erste Weltkongress für IVF in Helsinki stattfand, kam Edwards auf die Idee, eine europäische Fachgesellschaft für Reproduktionsmedizin zu gründen, die European Society of Human Reproduction and Embryology (ESHRE). Es wurde der jährlich stattfindende ESHREKongress ins Leben gerufen, der ein Jahr nach der Gründung 1985 erstmals in Bonn stattfand - mit 700 Teilnehmern. Damals konnten die Kongressteilnehmer noch einzeln per Handschlag begrüßt werden. Am ESHRE-Kongress 2010 in Rom nahmen über 10.000 Personen teil.

Edwards gründete die Zeitschriften Human Reproduction, Human Reproduction Update und Molecular Human Reproduction. Über viele Jahre war er ihr Herausgeber. Diese 3 Zeitschriften gehören nach wie vor zu den international führenden Zeitschriften in der Gynäkologie und Geburtshilfe.

Zusätzlich gründete er die Zeitschrift Reproductive Biomedicine online, die ebenfalls sehr erfolgreich im Bereich der Reproduktionsmedizin und Endokrinologie ist. Edwards genügte dies alles nicht. Er entwickelte immer neue Visionen, testete als Erster die Kryokonservierung überzähliger Embryonen, führte im Tierexperiment die erste Präimplantationsdiagnos- 
tik durch und hoffte, dass es möglich sei, diese Verfahren auch am Menschen zur Anwendungsreife zu führen. Darüber hinaus wendete er bereits 1965 erfolgreich die In-vitro-Maturation von Eizellen mit anschließender Befruchtung im Tiermodell an. Viele seiner Visionen wurden in die Tat umgesetzt.

Wir haben viele Kongresse und Workshops zusammen organisiert. Edwards war auch wissenschaftlicher Vater für viele deutsche Reproduktionsmediziner und Motor für die Reproduktionsmedizin in Europa. Es war für uns immer ein besonderes und inspirierendes Erlebnis, Zeit mit ihm zu verbringen. Als Dieter Krebs aus Bonn im Jahr 1992 den DGGG-Kongress in Berlin leitete, wurde Robert Geoffrey Edwards zum Ehrenmitglied der DGGG ernannt.

Das Editorial Board der Zeitschrift Gynäkologische Endokrinologie gratuliert ihm und sendet ihm herzliche Glückwünsche.

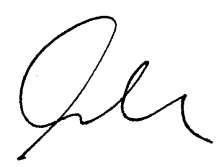

K. Diedrich

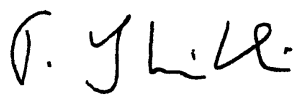

T. Strowitzki

\section{Korrespondenzadresse \\ Prof. Dr. K. Diedrich}

Klinik für Frauenheilkunde und Geburtshilfe, Universitätsklinikum Schleswig-Holstein, Campus Lübeck

Ratzeburger Allee 160, 23562 Lübeck

klaus.diedrich@uk-sh.de

\section{Prof. Dr. T. Strowitzki}

Abteilung für Gynäkologische Endokrinologie und Fertilitätsstörungen,

Universitäts-Frauenklinik

Voßstr. 9, 69115 Heidelberg

thomas.strowitzki@med.uni-heidelberg.de
F. Leidenberger, T. Strowitzki, O. Ortmann Klinische Endokrinologie für Frauenärzte

Heidelberg: Springer-Verlag 2009, 4., 778 S., 234 Abb., (ISBN 978-3-540-89759-0), gebunden, 189.95 EUR

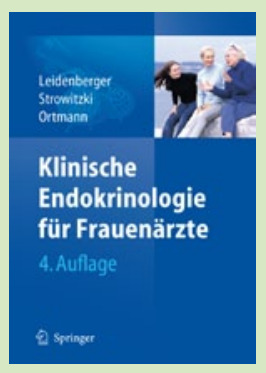

Die Gynäkologische Endokrinologie ist ein wesentlicher Teil der täglichen Arbeit eines jeden praktizierenden Gynäkologen. Dies steht im Widerspruch zu der gesundheitspoli-

tisch und ökonomisch vielleicht gewollten und zumindest gebilligten Verdrängung dieses Fachgebiets aus der universitären Landschaft. Trotzdem oder gerade deshalb werden Medien gefordert, die eine umfassende Information und Fortbildung eines angehenden und bereits praktizierenden Gynäkologen und gynäkologisch tätigen Endokrinologen ermöglichen.

Ein solches Medium ist zweifelsohne ein umfassendes Lehrbuch, das nicht nur das aktuelle Wissen leicht verständlich, klar und spannend aufbereitet, sondern auch die verschiedenen Aspekte und Bereiche des Faches themenübergreifend darstellt sowie praxisrelevant diagnostische und therapeutische Hilfestellungen gibt. Das Lehrbuch „Klinische Endokrinologie für Frauenärzte" erfüllt in meisterhafter Form diese Ansprüche und ist folglich im deutschen Sprachraum das große Standardwerk der Gynäkologischen Endokrinologie par excellence. Das Buch deckt nicht nur sämtliche Aspekte der Gynäkologischen Endokrinologie ab, sondern hält auch mit der kontinuierlichen Entwicklung in der Medizin Schritt.

Folglich war nach 4 Jahren eine Neuauflage dieses Buches eine logische Konsequenz dieser Ansprüche, um nicht nur der Leserschaft ein fundiertes, sondern auch ein stets hochaktuelles Wissen präsentieren zu können. In der 4. Auflage dieses renommierten Buches wurden alle Kapitel sorgfältig überarbeitet und dem zeitgemäßen Wissensstand angepasst. Neue Autoren konnten gewonnen werden, sodass viele Kapitel modifiziert und aufgefrischt wurden. Neu integriert wurde das Kapitel
„Endokrinologische Störungen an Haut und Haaren". Die Texte sind gut verständlich und werden durch aussagekräftige Abbildungen inhaltlich aufgewertet. Der Umfang des Buches und der damit verbundene Preis mögen zunächst verunsichern. Doch bei der Durchsicht wird klar, dass die übersichtliche Gliederung, die zum einen zügige Information zu allen relevanten Themen während der Sprechstunde erlaubt, zum anderen eine vertiefende und nachhaltige Weiterbildung nach der Sprechstunde ermöglicht, beides rechtfertigt. Nahezu keine Frage im gesamten Fachgebiet bleibt unbeantwortet. Dieses Buch dürfte und sollte somit bei jedem endokrinologisch interessierten Gynäkologen einen festen Platz in seiner täglichen Arbeit haben.

M. von Wolff(Bern) 